\title{
Metal Template Synthesis of Molecular Knots and Links
}

\author{
Jonathon E. Beves $\S^{\star}$ \\ §SCS-Metrohm Foundation Award for best oral presentation
}

\begin{abstract}
The use of metal ions as templates to direct the assembly of complex architectures and topologies is briefly reviewed, highlighting milestones in the field from [2]catenanes, through to trefoil knots, Solomon links, Borromean rings and most recently to a molecular pentafoil knot.
\end{abstract}

Keywords: Catenane $\cdot$ Knot · Link · Supramolecular · Topology

\section{Polymers and Molecular Materials: The Need for Topological Control?}

Interest in molecular materials has exploded in the past decade, with much emphasis on the fields of coordination networks (or MOFs), ${ }^{[1]}$ supramolecular gels $^{[2]}$ and supramolecular polymers. ${ }^{[3]}$ Applications of these new materials range from selective gas storage, ${ }^{[4]}$ small molecule sensing, catalysis, ${ }^{[5]}$ the development of self-healing polymers ${ }^{[6]}$ and tools for improved crystallisation in the pharmaceutical industry. ${ }^{[2]}$ However, control over the topology ${ }^{[1 a]}$ of coordination networks has been largely limited to the use of simple 'struts' and 'joins', to give (in varying degrees of reliability) $1 \mathrm{D},{ }^{[7]} 2 \mathrm{D}^{[8]}$ or $3 \mathrm{D}^{[9]}$ coordination networks with the most complex examples - such as an almost unbelievable 54-fold interpenetrated network ${ }^{[10]}$ - being the result of serendipity rather than design. Similarly, the synthesis of supramolecular gels $^{[2]}$ and polymers ${ }^{[3]}$ still largely rely on

diverse distributions of functionality and connectivities, rather than precise control over structure. It is well established that the topology of connectivities in extended materials can powerfully influence their bulk properties. For example, the presence of knots alters the strength of individual polymer strands, ${ }^{[11]}$ the position of crosslinks in supramolecular polymers has been shown to be critical to their properties, ${ }^{[12]}$ and the topology of linked colloids has allowed significant control over the properties of soft matter. ${ }^{[13]}$ However, chemists still remain relatively unable to control complex topologies in a predictable, welldefined manner. If materials are to be designed with specific molecular topologies - as opposed to simply being porous or forming gels - a greater degree of control over the structure of interwoven structures will be required.

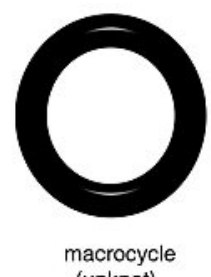
(unknot)

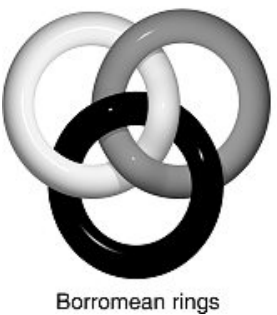

Borromean rings

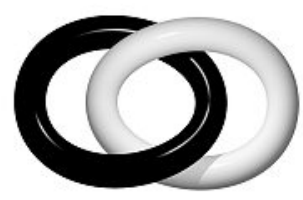

[2]catenane

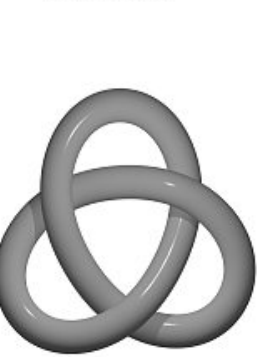

Trefoil knot
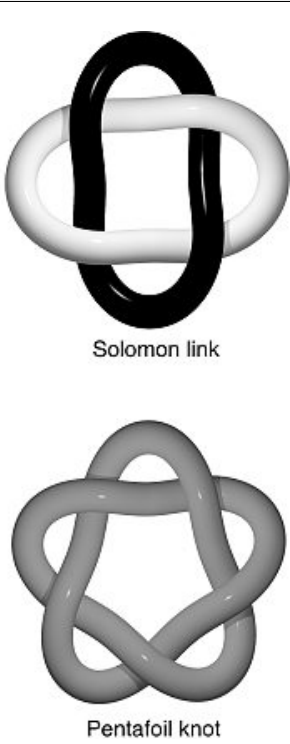

Fig. 1. Topological knots and links which have succumbed to small molecule synthesis. Note a simple macrocycle (known topologically as an 'unknot'), and all knots, including a trefoil knot and a pentafoil knot are topological isomers, differing only by the arrangement of their crossing points. 


\section{Metal Template Approaches to Interlocked Molecules}

The strategy[16] introduced by Sauvage was to employ a copper(I) centre as a tetrahedral template to gather and orient two bidentate ligands (phenantholine-derivatives, Scheme 1a) appropriately in space to generate a 'crossing point'. [15] The next challenge was to react the end-groups of the ligands together in the desired manner, avoiding both intermolecular (which would in this case give oligomeric or polymeric structures) and inter-ligand reactions (which would give a topologically uninteresting macrocycle, also known as the 'unknot'). Reaction of the phenol end-groups of this crossing point with a suitable diiodo linker (using Williamson ether synthesis) gave the first high-yield synthesis of a mechanically interlocked [2]catenane (Scheme 1a). Almost two decades later, the Leigh group applied metaltemplate imine-bond formation to prepare [2]catenanes about a range of octahedral metal ions using 2,6-diformylpyridine and terminal diamino linkers (Scheme 1b). [17] This strategy has the advantage of the imine bond forming reaction being reversible, leading to the potential for the assembly to occur under thermodynamic control and subsequently deliver high yields. Importantly, subsequent reduction of the imine groups to kinetically inert amines allowed the [2]catenane to be 'locked'. Imine bond formation was also used by the Nitschke group, then based in Geneva, to form [2]catenanes using two copper(I) centres, ${ }^{[18]}$ and remains the central tool in their continuing research program.

\section{The First Non-Trivial Synthetic Molecular Knot: The Trefoil Knot}

In 1989 the first synthetic molecular knot - a trefoil knot, see Fig. 1 for a cartoon - was prepared using an extension of Sauvage's original strategy.[19] A ligand with two phenanthroline binding groups separated by a short spacer (to prevent the formation of a 2:1 ligand : metal complex) was reacted with copper(I) ions to form a dimetallic, double-stranded helicate from two copper(I) ions and two organic ligands. Williamson ether synthesis formed the trefoil knot in low yield $(\sim 2 \%)$, which was later increased to a respectable $29 \%$ by the use of a rigid meta-substituted phenyl spacer which helped stabilise the entwined assembly. However, it was the introduction of efficient catalysts for ring-closing olefin metathesis (RCM) which allowed the knot to be synthesised in an impressive $74 \%$ yield (Scheme 1c). Octahedral iron(II) ions have also been exploited to template the formation of a trefoil knot using linked terpyridine-based ligands, although the yield was significantly lower (20\%). ${ }^{[20]}$ Hunter and co-workers recently reported the use of a single tris(2,2'-bipyridine) ligand which was capable of being wrapped around an octahedral zinc(II) ion to form a trefoil knot in $68 \%$ yield. ${ }^{[21]}$ The use of a single metal ion to produce multiple crossing points suggests more complex knots may be accessed via similarly well-designed methods. It is worth noting that this result comes a decade after the pre-cursor acyclic complex was reported, ${ }^{[22]}$ highlighting the difficulties often present when attempting to 'tie the knot'. The smallest trefoil

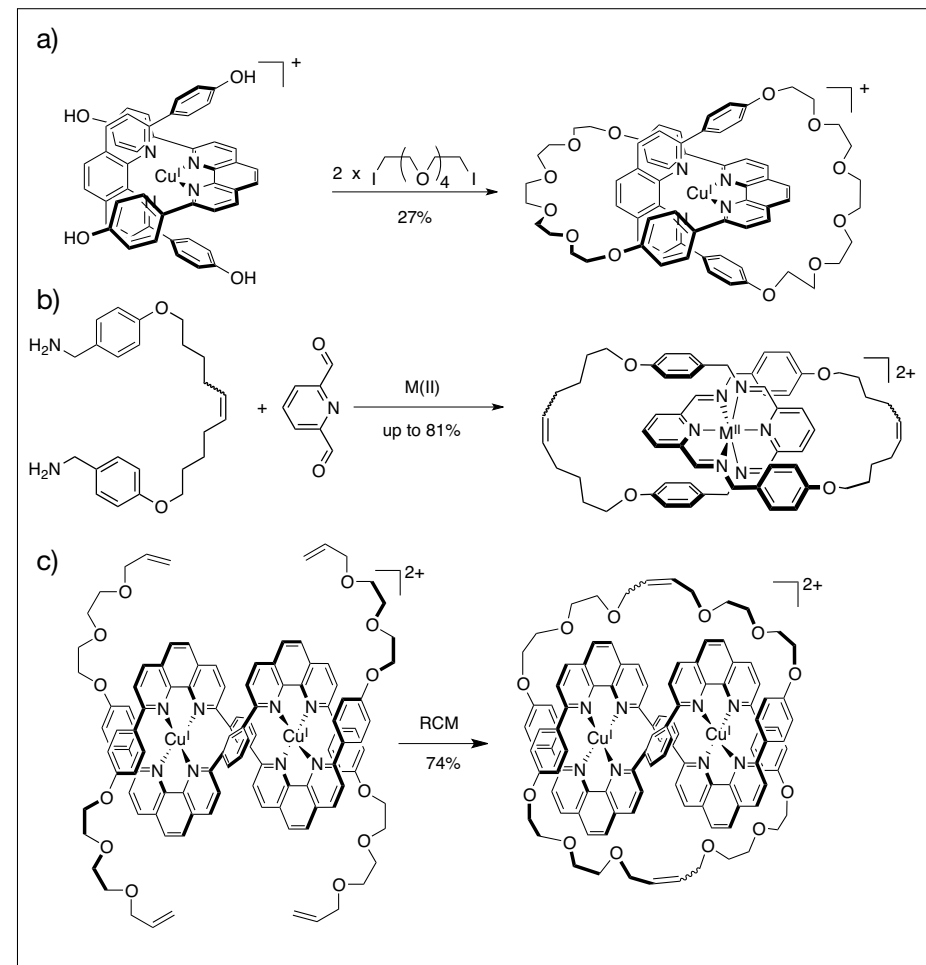

Scheme 1. Metal template synthesis of small molecule links and knots, showing several key developments. a) Synthesis of the first metal template catenane, which used a tetrahedral copper(I) template to arrange phenol groups in space for Williamson ether formation. ${ }^{[15]}$ b) The use of octahedral metal ions and reversible imine bond formation to form [2]catenanes under thermodynamic control. ${ }^{[17]} \mathrm{c}$ ) Synthesis of an early molecular trefoil knot using multiple metal centres to generate the required crossing points, and ring-closing olefin metathesis (RCM) to give a high yield. ${ }^{[19]}$ knot to date, ${ }^{[23]}$ with an organic backbone of 76 atoms, was also recently synthesised by the Leigh group using an active-metal template approach. Beginning with a single ligand strand, a copper(I) ion acted as a template to form the crossing points, and a separate copper(I) ion subsequently as a catalyst for the azide-alkyne cycloaddition ('click') reaction within a defined cavity, to give the knotted architecture.

\section{Knots and Chirality}

Knots are inherently chiral species, and chiral HPLC has been used to separate the two enantiomers of Sauvage's trefoil knot. ${ }^{[24]}$ Through a collaborative effort between Sauvage and von Zelewsky, who was based in Fribourg, an elegant approach allowed a single enantiomer to be specifically formed. ${ }^{[25]}$ Chiral groups were fused to the ends of the ligand to strictly control the stereochemistry of the metal centres and deliver an enantiopure molecular trefoil knot in $74 \%$ yield. ${ }^{[25]}$ This degree of control of the stereochemistry of large assemblies has potential applications in the development of enantioselective receptors or catalysts.

\section{Higher Order Molecular Links: Solomon Links}

Sauvage and co-workers extended their successful strategy to form linear helicates with three copper(I) centres, reaching, what appeared to be, the furthest this strategy could be stretched. In order to successfully form the desired structure, a topological constraint ${ }^{[16]}$ was required where one ring was pre-formed so only a single (as opposed to double) ring-closure reaction would be required. Williamson ether synthesis then gave the doubly interlocked catenane (see Fig. 1 for a cartoon), known as a Solomon link (or a Solomon Knot, although it is not technically a knot). The metal centres were removed, and the resulting free doubly interlocked catenane was isolated in $2 \%$ yield. An alternative approach was to form a precursor helicate with three lithium(I) ions and to use RCM for the ring closure, which gave a Solomon link in 30\% yield. Other examples of Solomon links have followed, including those formed from palladium(II)-containing metallocycles, ${ }^{[26]}$ and an example related to the Borromean rings discussed below. ${ }^{[27]}$

\section{Higher Order Molecular Links: Borromean Rings}

Borromean rings are a topology formed with three separate rings, where no two 


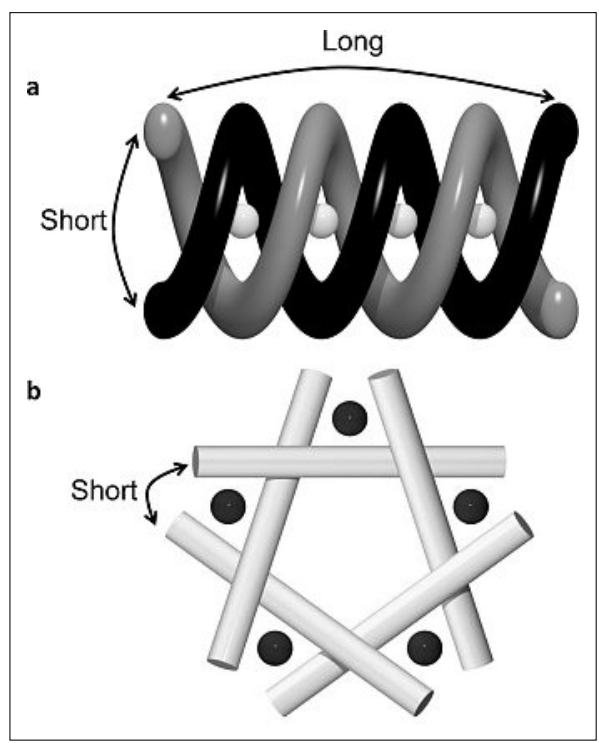

Fig. 2. Two strategies to form a pentafoil knot. a) The use of a linear helicate easily generates the five required crossing points, but the final ring closure to give a pentafoil knot must occur over a long distance, with the dominant competing reaction (which gives a simple macrocycle) involving only a short distance. b) The use of a cyclic structure allows the desired ring closure to be only a very short distance, with the sacrifice being that five ring closing reactions will be required, as opposed to just two for the linear example.

rings are interlocked, but the overall entanglement cannot be disassembled without the breaking of at least one ring (see Fig. 1. for a cartoon representation). Borromean rings with metal-organic connections were formed serendipitously by Schröder, Champness and co-workers, where six polypyridyl ligands were combined with six silver(I) or copper(I) ions to produce the interwoven structure. However, it is Stoddart and co-workers use of zinc(II) template imine bonds in a remarkably robust one-pot which stands alone as the most impressive synthetic achievement in the field. ${ }^{[28]}$ In this assembly eighteen individual components spontaneously assemble into the desired link in $90 \%$ iso- lated yield (quantitative by NMR). This synthesis is sufficiently straightforward that it has been shown to be suitable for an undergraduate laboratory![29] Surprisingly, although the use of copper(II) also generated the same topology, the use of mixed copper(II) and zinc(II) gave a Solomon link - again demonstrating the subtleties involved in these assembly processes.

\section{The Second Non-Trivial Synthetic Molecular Knot: A Pentafoil Knot}

Attempts to further extend the Sauvage linear helicate strategy to higher order knots and links have been reported.[30] These efforts proved unsuccessful, presumably due to the long spatial separation of the desired end groups to be reacted, compared with the relatively short distance between those which would give a simple macrocycle (Fig. 2a). Inspired by Lehn's seminal work with circular helicates formed with iron(II) salts, ${ }^{[31]}$ we recently reported ${ }^{[32]}$ an alternative strategy (Fig. 2b) to form a pentafoil knot. By using a cyclic structure as a scaffold the distance between the 'ends' to be reacted is short, increasing the probability of the desired reactions. The downside is that five such reactions will now be required, as opposed to only two where a linear helicate is used - increasing the possibility for errors. Lehn's original ligand was modified to introduce imine bonds to allow a capacity for error correction during the ring closure reactions. The use of a range of iron(II) salts and diamine linkers gave only polymeric material, but iron(II) chloride and a diamine with a glycol spacer (Fig. 3) was found to produce the desired knot. This demanding assembly requires the subtle energy gain delivered by the gauche effect of the glycol linkers, which allow the linker to form a low energy turn, as later evidenced in the solid-state structure. ${ }^{[32]}$ The formation of this knot represents the first new molecular primary knot in over 20 years.

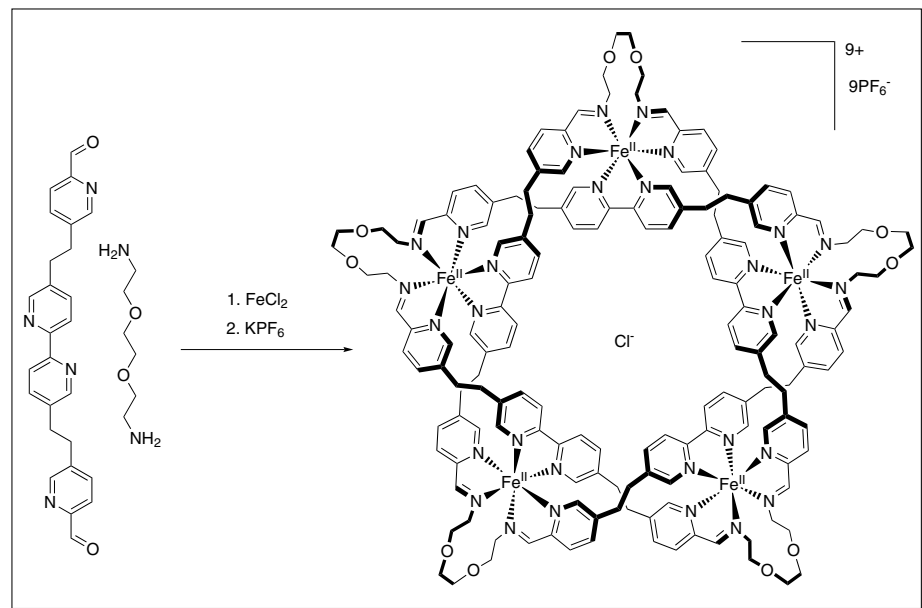

Fig. 3. Synthesis of the first pentafoil knot, using iron(II) ions to direct the imine bond formation between a dialdhyde and a dimaine linker. ${ }^{[32]}$

\section{A Future for Topological Chemistry?}

Far from being dead, the success of new approaches to preparing entwined and interlocked molecular structures offers inspiration for the development of increasingly complex architectures, unimaginable only a few years ago. However, future goals will not merely represent a 'stamp collection' of new topologies, but the demonstration that these structures - with their well-expressed chirality, potentially useful cavities and versatility of functionalisation - may be exploited to form molecular devices or machines. Additionally, the lessons learnt controlling the topology of small molecules should be applied to preparing molecular materials, allowing manipulation of bulk properties by precise control of the topology of extended structures to form lighter, stronger and smarter functional materials.

\section{Acknowledgement}

The Swiss National Science Foundation is acknowledged for their generous award of a Post-Doctoral Fellowship to the author. Prof David A. Leigh is thanked for his guidance and the University of Edinburgh and the EPSRC are acknowledged for financial support.

Received: January 23, 2012

[1] a) S. R. Batten, R. Robson, Angew. Chem. Int. Ed. 1998, 37, 1461; b) O. M. Yaghi, M O'Keeffe, N. W. Ockwig, H. K. Chae, M. Eddaoudi, J. Kim, Nature 2003, 423, 705.

[2] J. A. Foster, M.-O. M. Piepenbrock, G. O Lloyd, N. Clarke, J. A. K. Howard, J. W. Steed, Nature Chem. 2010, 2, 1037.

[3] T. F. A. de Greef, E. W. Meijer, Nature 2008 453, 171.

[4] J. R. Li, R. J. Kuppler, H. C. Zhou, Chem. Soc Rev. 2009, 38, 1477.

[5] a) J. Lee, O. K. Farha, J. Roberts, K. A. Scheidt, S. T. Nguyen, J. T. Hupp, Chem. Soc. Rev. 2009, 38, 1450; b) C. D. Wu, A. Hu, L. Zhang, W. B. Lin, J. Am. Chem. Soc. 2005, 127, 8940.

[6] M. Burnworth, L. Tang, J. R. Kumpfer, A. J. Duncan, F. L. Beyer, G. L. Fiore, S. J. Rowan, C. Weder, Nature 2011, 472, 334.

[7] a) J. E. Beves, E. C. Constable, C. E. Housecroft, C. J. Kepert, M. Neuburger, D. J. Price, S. Schaffner, J. A. Zampese, Dalton Trans. 2008, 6742; b) J. E. Beves, E. C. Constable, C. E. Housecroft, C. J. Kepert, D. J. Price, CrystEngComm 2007, 9, 456; c) J. Ehrhart, J.-M. Planeix, N. Kyritsakas-Gruber, M. W. Hosseini, Dalton Trans. 2009, 2552.

[8] a) J. L. Sagué, K. M. Fromm, Cryst. Grow. Des. 2006, 6, 1566; b) J. E. Beves, E. C. Constable, S. Decurtins, E. L. Dunphy, C. E. Housecroft, T. D. Keene, M. Neuburger, S. Schaffner, CrystEngComm 2008, 10, 986.

[9] a) V. A. Blatov, L. Carlucci, G. Ciani, D. M. Proserpio, CrystEngComm 2004, 6, 377; b) Y. M. A. Yamada, Y. Maeda, Y. Uozumi, Org. Lett. 2006, 8,4259 .

[10] H. Wu, J. Yang, Z.-M. Su, S. R. Batten, J.-F. Ma, J. Am. Chem. Soc. 2011, 133, 11406.

[11] A. M. Saitta, P. D. Soper, E. Wasserman, M. L. Klein, Nature 1999, 399, 46.

[12] U. Rauwald, O. A. Scherman, Angew. Chem. Int. Ed. 2008, 47, 3950 
[13] U. Tkalec, M. Ravnik, S. Copar, S. Zumer, I. Musevic, Science 2011, 333, 62.

[14] a) H. L. Frisch, E. Wasserman, J. Am. Chem. Soc. 1961, 83; b) J. S. Siegel, Science 2004, $304,1256$.

[15] C. O. Dietrich-Buchecker, J.-P. Sauvage, J.-M Kern, J. Am. Chem. Soc. 1984, 106, 3043.

[16] J. E. Beves, B. A. Blight, C. J. Campbell, D. A. Leigh, R. T. McBurney, Angew. Chem. Int. Ed. 2011, 50, 9260.

[17] D. A. Leigh, P. J. Lusby, S. J. Teat, A. J. Wilson, J. K. Y. Wong, Angew. Chem. Int. Ed. 2001, 40, 1538.

[18] M. Hutin, C. A. Schalley, G. Bernardinelli, J. R. Nitschke, Chem.-Eur. J. 2006, 12, 4069.

[19] C. O. Dietrich-Buchecker, J.-P. Sauvage, Angew. Chem. Int. Ed. Engl. 1989, 28, 189.
[20] G. Rapenne, C. Dietrich-Buchecker, J.-P. Sauvage, J. Am. Chem. Soc. 1999, 121, 994.

[21] J. Guo, P. C. Mayers, G. A. Breault, C. A. Hunter, Nature Chem. 2010, 2, 218.

[22] H. Adams, E. Ashworth, G. A. Breault, J. Guo, C. A. Hunter, P. C. Mayers, Nature 2001, 411, 763.

[23] P. E. Barran, H. L. Cole, S. M. Goldup, D. A. Leigh, P. R. McGonigal, M. D. Symes, J. Wu, M. Zengerle, Angew. Chem. Int. Ed. 2011, 50, 12280 .

[24] G. Rapenne, C. Dietrich-Buchecker, J.-P. Sauvage, J. Am. Chem. Soc. 1996, 118, 10932.

[25] L.-E. Perret-Aebi, A. von Zelewsky, C. DietrichBuchecker, J.-P. Sauvage, Angew. Chem. Int. Ed. 2004, 43, 4482.

[26] C. Peinador, V. Blanco, J. M. Quintela, J. Am. Chem. Soc. 2008, 131, 920 .
[27] C. D. Pentecost, K. S. Chichak, A. J. Peters, G. W. V. Cave, S. J. Cantrill, J. F. Stoddart, Angew. Chem. Int. Ed. 2007, 46, 218.

[28] K. S. Chichak, S. J. Cantrill, A. R. Pease, S. H. Chiu, G. W. V. Cave, J. L. Atwood, J. F. Stoddart, Science 2004, 304, 763.

[29] C. D. Pentecost, N. Tangchaivang, S. J. Cantrill, K. S. Chichak, A. J. Peters, J. F. Stoddart, J. Chem. Ed. 2007, 84, 855.

[30] C. Dietrich-Buchecker, B. Colasson, D. Jouvenot, J.-P. Sauvage, Chem.-Eur. J. 2005, $11,4374$.

[31] B. Hasenknopf, J.-M. Lehn, B. O. Kneisel, G Baum, D. Fenske, Angew. Chem. Int. Ed. Engl. 1996, 35, 1838 .

[32] J.-A. Ayme, J. E. Beves, D. A. Leigh, R. T. McBurney, K. Rissanen, D. Schultz, Nature Chem. 2012, 4, 15 . 